\title{
Effects of Antibiotic Therapy in Primary Sclerosing Cholangitis with and without Inflammatory Bowel Disease: A Systematic Review and Meta-Analysis
}

\author{
Ayesha Shah, MBBS, FRACP1,2,3,* Darrel Crawford, MBBS, MD, FRACP ${ }^{1,4}$ Daniel Burger, MBBS, FRACP ${ }^{1,2}$ \\ Neal Martin, MBBS, FRACP ${ }^{1,2}$ Marjorie Walker, MBBS, FRCPath, FRCPA, AGAF ${ }^{5, *}$ \\ Nicholas J. Talley, MD, PhD, FAHMS, FRACP ${ }^{5, *}$ Caroline Tallis, MBBS, FRACP ${ }^{1,2}$ \\ Michael Jones, BSC, PhD ${ }^{6, *}$ Katherine Stuart, MBBS, PhD, FRACP ${ }^{1,2}$ Simon Keely, MBBS, PhD \\ Peter Lewindon, MBBS, FRACP, FRCP ${ }^{1,7}$ Graeme A. Macdonald, MBBS, FRACP, PhD ${ }^{1,2}$ \\ Mark Morrison, $\mathrm{PhD}^{8}$ Gerald J. Holtmann, MD, PhD, MBA, FRACP, FRCP, FAHMS ${ }^{1,2,3, *}$
}

\footnotetext{
${ }^{1}$ Faculty of Medicine, The University of Queensland, Brisbane, QLD, Australia

${ }^{2}$ Department of Gastroenterology \& Hepatology, Princess Alexandra Hospital, Brisbane, QLD, Australia

${ }^{3}$ Translational Research Institute, Princess Alexandra Hospital, Brisbane, QLD, Australia

${ }^{4}$ Gallipoli Research Foundation, Brisbane, QLD Australia

${ }^{5}$ Faculty of Health and Medicine, University of Newcastle, Newcastle, NSW Australia

${ }^{6}$ Department of Psychology, Macquarie University, Sydney, NSW, Australia

${ }^{7}$ Lady Cilento Children's Hospital, South Brisbane, QLD, Australia

${ }^{8}$ Department of Microbial Biology and Metagenomics, Diamantina Institute, University of Queensland, Woolloongabba, Brisbane, QLD, Australia
}
Address for correspondence Gerald J. Holtmann, MD, PhD, MBA, FRACP, FRCP, FAHMS, Department of Gastroenterology and Hepatology, Princess Alexandra Hospital, Brisbane, and University of Queensland, Ipswich Road, Woolloongabba, QLD 4102, Australia (e-mail: g.holtmann@uq.edu.au).

Semin Liver Dis 2019;39:432-441.

Abstract
Keywords
- vancomycin
- antibiotics
- primary sclerosing
cholangitis
- inflammatory bowel
disease
- ulcerative colitis
- Crohn's disease
- microbiome

The authors conducted a systematic review and meta-analysis to assess the effect of antibiotic therapy in primary sclerosing cholangitis (PSC). Effect of antibiotic therapy on Mayo PSC Risk Score (MRS), serum alkaline phosphatase (ALP), total serum bilirubin (TSB), and adverse events (AEs) rates were calculated and expressed as standardized difference of means or proportions. Five studies including 124 PSC patients who received antibiotics were included. Overall, antibiotic treatment was associated with a statistically significant reduction in ALP, MRS, and TSB by $33.2,36.1$, and $28.8 \%$, respectively. ALP reduction was greatest for vancomycin $(65.6 \%, p<0.002)$ and smallest with metronidazole $(22.7 \%, p=0.18)$. Overall, $8.9 \%$ (95\% confidence interval: 3.9-13.9) of patients had AEs severe enough to discontinue antibiotic therapy. In PSC patients, antibiotic treatment results in a significant improvement in markers of cholestasis and MRS. Antibiotics, particularly vancomycin, may have a positive effect on PSC either via direct effects on the microbiome or via host-mediated mechanisms.
Primary sclerosing cholangitis (PSC) is an immunemediated chronic cholestatic liver disease of unknown etiology, characterized by ongoing inflammation, destruc-

* for the Australian Gastrointestinal Research Alliance (AGIRA). tion, and subsequent fibrosis of intra- and extrahepatic bile ducts. ${ }^{1}$ PSC is progressive, leading to fibrosis, liver cirrhosis, portal hypertension, hepatic decompensation, and an increased risk of malignancy in the majority of patients. $^{2-4}$ published online

July 17, 2019

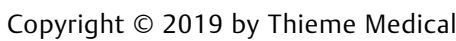
Publishers, Inc., 333 Seventh Avenue, New York, NY 10001, USA. Tel: +1(212) 584-4662.
DOI https://doi.org/ 10.1055/s-0039-1688501. ISSN 0272-8087. 
Considered a rare disease, the incidence and prevalence ranges from approximately 0.5 to 1.3 cases per 100,000 person-years and 3.85 to 16.2 cases per 100,000 personyears, respectively. ${ }^{5-7}$ There is a strong association between PSC and inflammatory bowel disease (IBD), particularly ulcerative colitis (UC), ${ }^{8,9}$ and between 60 and $70 \%$ of patients with PSC have IBD. ${ }^{10}$

Due to the progressive chronic liver disease and subsequent liver failure, liver transplantation is often required in patients with PSC, ${ }^{11-13}$ and median life expectancy after diagnosis of PSC ranges from 13 to 21 years ${ }^{14}$ without liver transplantation. After liver transplantation, PSC recurs in 8.6 to $27 \%$ of patients, ${ }^{15}$ emphasizing the need for more efficacious therapies in these patients. Thus far, there is lack of evidence that any drug treatment (immunosuppressive, antiinflammatory, and antifibrotic agents) alters the natural course of the disease. ${ }^{1,11-13,16-18}$

The pathophysiology of PSC is incompletely understood, but the process is likely multifactorial. The epithelial cells lining the bile ducts (i.e., cholangiocytes) are now thought to be not only a target of injury in PSC but also actively involved drivers in the course of the disease. ${ }^{19,20}$ PSC likely occurs in genetically susceptible individuals, perhaps after exposure to environmental triggers. ${ }^{11,13}$ Alterations of the gastrointestinal microbiome and gut permeability occurs, referred to as "PSC microbiota" or the "leaky gut" hypothesis, as supported by several observations from preclinical $^{21-23}$ and clinical studies. ${ }^{24}$ The altered gut microbiome also may result in alterations of the bile acid pool, ${ }^{25}$ which potentially also plays a role in the pathophysiology of PSC. ${ }^{26}$ However, the observed alterations of the gastrointestinal microbiome might be a consequence rather than the cause of the disease. There is now some evidence that the course of the disease is modified by antibiotic therapies. In 1959, Rankin et al ${ }^{17}$ successfully used tetracycline $(500 \mathrm{mg}$ once daily for 10 months) to improve biochemical and clinical measures in five patients with chronic, progressive pericholangitis and associated UC likely caused by portal bacteremia. During the past 10 years, several case reports, case series, and clinical trials in children ${ }^{27-29}$ and adults ${ }^{28,30-38}$ have explored various antimicrobial agents for PSC patients. In these studies, antibiotics appeared to improve serum alkaline phosphatase (ALP) levels ${ }^{39}$ and the PSC Mayo Risk Score (MRS). ${ }^{40}$

To examine the evidence for the therapeutic efficacy of (oral) antibiotics for the treatment of PSC, we performed a systematic review of all clinical trials that have evaluated antibiotics in adult patients with PSC. We aimed to assess (1) change from baseline in MRS and the markers of cholestasis (ALP) and total serum postantibiotic (or placebo) therapy and (2) AEs leading to discontinuation of antibiotic treatment.

\section{Materials and Methods}

\section{Search Strategy}

Electronic databases, including PUBMED, MEDLINE (OvidSP), and EMBASE, were searched from initiation up to June 2018 for all studies assessing the use of antibiotics in PSC patients with or without IBD. This was supplemented by "snowball" methods such as manual searches of the bibliographies of relevant papers, specialist journals, to identify all relevant studies. The search strategy included ['PSC' OR 'primary sclerosing cholangitis' OR 'cholangitis'] AND [('IBD' OR 'inflammatory bowel disease' OR 'UC' OR 'ulcerative colitis' OR CD OR 'Crohn's disease')]. These were combined using the set operator AND with studies identified with the terms: "antibiotics or antimicrobial agents" and the following freetext terms: vancomycin, metronidazole, tetracycline, doxycycline, gentamycin, tetracycline, minocycline, azithromycin, rifaximin, sulfasalazine, cephalosporins, and penicillin. The search was not limited by language to ensure that all appropriate papers were captured. All manuscripts were required to have an English abstract. The detailed search strategy was developed in consultation with a research librarian and the results of the literature search are outlined in -Fig. 1.

\section{Outcome Assessment}

The primary outcomes assessed were the change from baseline in ALP, total serum bilirubin levels, and MRS postantibiotic (or placebo) treatment. Secondary outcomes included drug-related adverse events (AEs) leading to discontinuation of antibiotic treatment.

\section{Selection of Studies}

Two authors (G.H. and A.S.) independently conducted an initial screening of identified abstracts and titles. Abstracts were eliminated in this initial screening if they were case reports or case series, animal studies, or if they did not investigate the association between antibiotics and PSC with or without coexisting IBD. Full texts of the remaining articles were retrieved and reviewed. Articles were considered for inclusion only if they reported original data from single arm open-labeled studies or randomized controlled trials (RCTs), reporting use of antimicrobial agents for a minimum duration of 12 weeks in treatment of patients with an established diagnosis of PSC, and the manuscript or abstracts were published in peer-reviewed journals. The diagnosis of PSC was established by the following criteria: ALP greater than 1.5 times the upper limit of normal for at least 6 months with cholangiographic (e.g., magnetic resonance cholangiography, endoscopic retrograde cholangiography, percutaneous transhepatic cholangiography) evidence of characteristic bile duct changes with multifocal strictures and segmental dilatations, and exclusion of secondary causes of sclerosing cholangitis. Detailed eligibility criteria for study inclusion are provided in - Table 1. Disagreements between reviewers were resolved by mutual consensus after reference to the original published paper.

\section{Data Extraction and Quality Assessment}

All data were extracted independently by two reviewers onto a Microsoft Excel spreadsheet (Office 2016 professional edition; Microsoft Corp.), with disagreements resolved by consensus. For each trial, pre- and post-treatment values for ALP, serum total bilirubin, C-reactive protein, and MRS were extracted. In addition, the following information was 


\section{Study Design}

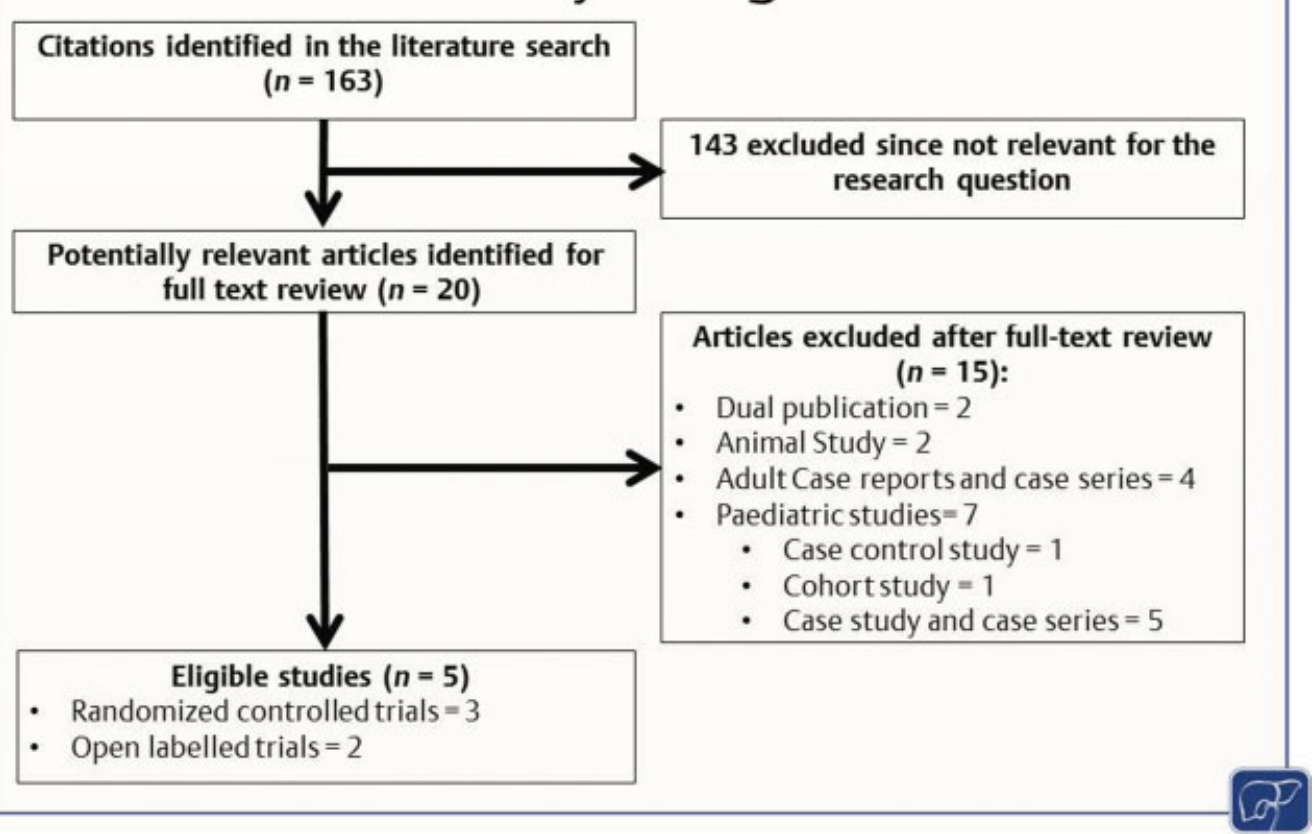

Fig. 1 Flow diagram showing results of literature search.

extracted from each trial: author, year of publication, study design, country of origin, proportion of male patients, proportion of patients with coexisting IBD, mean age, dose, type and duration of antibiotic therapy, concurrent use of other medications, total number of reported AEs, total number of AEs leading to discontinuation of antibiotic therapy, clinical criteria for diagnosis of PSC, and definition of primary and secondary outcomes.

The current systematic review and meta-analysis was consistent with the proposals for the reporting of meta-analysis of observational studies in epidemiology (Meta-analysis of Observational Studies in Epidemiology $)^{41}$ and met the preferred reporting items for systematic reviews and meta-analysis statement requirements (see - Supplementary Material, online only). ${ }^{42}$

\section{Data Analysis}

In an initial step, changes from the baseline values in ALP, MRS, total bilirubin postantibiotic treatment, or placebo were as calculated. In a second step, the pooled difference in means and 95\% confidence intervals (CIs) for the primary outcome measures (ALP, MRS, and total bilirubin) in PSC patients receiving antibiotic therapy (or placebo) were separately determined as described below. Subgroup analyses according to type of antibiotic therapy (vancomycin, metronidazole, minocycline, rifaximin) on each primary outcome measures were also performed. Pooled discontinuation rates due to AEs were calculated. Proportions and 95\% CI were calculated when appropriate.

Analyses were performed utilizing the Comprehensive Meta-analysis (CMA), Version 3.3.070 (Biostat, Inc.). Pooled estimates of effect of antibiotic therapy on primary outcome measures were calculated using a random effects model ${ }^{43}$ as all tests of between-study homogeneity indicated that variation between individual study estimates was consistent with random sampling variation. Between-study variation was evaluated using Cochrane's test ${ }^{44}$ and was quantified through the $\mathrm{I}^{2}$ index in which values close to 100 indicate substantial variation between studies while values close to zero indicate minimal between-study variation. The statistical package CMA

Table 1 Eligibility criteria for the studies included in systematic review and meta-analysis

\begin{tabular}{|l|}
\hline Eligibility criteria \\
\hline - Adults (participants aged $>16 \mathrm{y}$ ) \\
\hline - Randomized controlled trials or open-labeled trials \\
\hline - Patients with established diagnosis of PSC (utilizing clinically validated methods ${ }^{\text {a }}$ ), treated with antibiotics \\
\hline - Minimum duration of therapy $12 \mathrm{wk}$ \\
\hline
\end{tabular}

Abbreviation: ALP, alkaline phosphatase; PSC, primary sclerosing cholangitis.

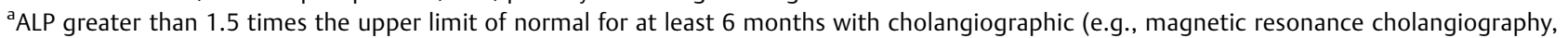
endoscopic retrograde cholangiography, percutaneous transhepatic cholangiography) evidence of characteristic bile duct changes with multifocal strictures and segmental dilatations, and exclusion of secondary causes of sclerosing cholangitis. 
uses logit transformation of proportions and the variance of the logit to estimate pooled event rates within groups and to compare event rates between groups. If one or more cells had a value of 0 , then the CMA software automatically adds a fixed value of 0.5 to all the cells of the study results tables where the problems occur for computation of log odds ratio and variance. Further, either chi-square test, $p$-value of $<0.10$, or $\mathrm{I}^{2}>50 \%$ indicated substantial heterogeneity.

\section{Results}

The initial literature search revealed 163 publications. Of these, 20 published articles appeared to be relevant for the study question and were retrieved for further evaluation. Fifteen articles were excluded for not fulfilling the inclusion criteria leaving five eligible studies ( - Fig. 1). Three of the five studies were RCTs ${ }^{35-37}$ and two were open-labeled studies. $^{34,38}$ Two RCTs compared the efficacy of antibiotics (vancomycin $^{37}$ and metronidazole ${ }^{35}$ ) against placebo in PSC patients, while one nonplacebo-controlled $\mathrm{RCT}^{36}$ compared the efficacy of low and high doses of vancomycin and metronidazole in PSC patients. One open-labeled trial used rifaximin $^{38}$ and one used minocycline. ${ }^{34}$ Three of the five studies were conducted in the U.S. ${ }^{34,36,38}$ and the remaining two were from Iran ${ }^{37}$ and Finland. ${ }^{35}$ The primary and secondary outcomes and patient characteristics of the studies included in the systematic review and meta-analysis are outlined in - Tables 2 and 3, respectively.

\section{Effects of Placebo on Outcome Parameters}

Two studies utilizing metronidazole for 36 months ${ }^{35}$ and vancomycin for 12 weeks ${ }^{37}$ included placebo arms. During placebo treatment, no significant improvements of ALP, total serum bilirubin, and MRS were observed (-Table 2 ).

\section{Effect of Antibiotic Treatment on Primary Outcome Measure in PSC Patients}

A total of 124 PSC patients (98 [79\%], with concurrent IBD) received antibiotic therapy ( 57 treated with metronidazole, 35 with vancomycin, 16 each with rifaximin and minocycline). Treatment with antibiotics in PSC patients was associated with a statistically significant reduction in ALP, MRS, and total serum bilirubin level by $33.2,36.1$, and $28.8 \%$, respectively (-Figs. 2-4). Moderate heterogeneity was detected between those studies reporting ALP $\left(\mathrm{I}_{2}=44.93, p=0.08\right)$ and MRS $\left(\mathrm{I}_{2}=47.60, p=0.06\right)$. In contrast, only minimal heterogeneity was detected between those studies reporting change in total serum bilirubin level $\left(\mathrm{I}_{2}=20.02, p=0.23\right)$.

\section{Effects of Different Antibiotics}

Alkaline phosphatase reduction was greatest with vancomy$\operatorname{cin}(65.6 \%, p<0.002)$ and smallest for metronidazole $(22.7 \%$, $p=0.18$ ). Both antibiotics had similar effects on MRS (46.8 and $46.6 \%$ by vancomycin and metronidazole, respectively, all $p<0.05)$, while vancomycin $(38.6 \%, p=0.12)$ was not significantly better than metronidazole $(34.2 \%, p=0.07)$ in decreasing total bilirubin level. A single open-labeled study utilizing minocycline in 16 PSC patients had significant effects on ALP, MRS, and total serum bilirubin levels (reduction by $56.2 \%, p=0.03 ; 53.3 \%, p=0.05$; and $42.5 \%, p=0.10$, respectively). Treatment with rifaximin did not result in an improvement of ALP, total serum bilirubin, or MRS.

\section{Adverse Events with Antibiotic (Placebo) Treatment}

All five trials reported on AEs in PSC patients that lead to discontinuation of treatment. Overall, $8.9 \%$ (95\% CI: 3.913.9) of patients had AEs severe enough to discontinue antibiotic therapy. Placebo did not result in discontinuation of therapy due to AEs (-Table 2). Among antibiotics, the incidence of AEs was smallest for vancomycin (5.7\%) and greatest for rifaximin (19\%, n.s.).

\section{Effect of Antibiotic Therapy on IBD Associated with PSC}

Only one study ${ }^{37}$ specifically assessed the effect of antibiotic therapy on IBD-related symptoms. Twelve weeks after initiation of treatment with vancomycin as compared with placebo, they found significant differences in the improvement in IBD-related symptoms (diarrhea, blood in stools, and abdominal pain, nausea, and vomiting). The remaining studies did not report any further IBD activity parameters. Thus, no further analysis of the effect of antibiotics on the IBD activity could be conducted.

\section{Discussion}

Primary sclerosing cholangitis is a rare disease, and within 8 to 12 years of diagnosis patients die or require liver transplantation. ${ }^{45}$ The low incidence and prevalence is a barrier for large RCTs. However, case series and, more recently, small RCTs have evaluated the efficacy of antibiotics as a therapeutic option in PSC. This is the first systematic review and meta-analysis on the effects of antibiotics in adult patients with PSC. While limited to cohort studies and small RCTs, the data strongly suggest that at least some antibiotics improve markers of cholestasis and MRS and thus have the potential to modify the course of disease in patients with PSC.

This systematic review and meta-analyses applied strict criteria and includes only five studies conducted in three countries with a sample size of 124 PSC patients, where $79 \%$ had coexisting IBD. Overall, antibiotic therapy significantly improved ALP and MRS. Vancomycin appeared to be the most effective antibiotic with the best safety profile. Vancomycin treatment specifically targets Gram-positive bacteria, ${ }^{46}$ which includes various Clostridium spp. known to be primarily involved with the dehydroxylation of primary bile acids, into the secondary bile acids present in the distal small intestine and colon. ${ }^{47,48}$ Secondary bile acids are highly hydrophobic and toxic, and increased concentrations in the liver have been linked to inflammation, cholestasis, gallstone formation, ${ }^{49}$ and carcinogenesis. ${ }^{50}$ Thus, it could be speculated that antibiotic therapy influences bile acid metabolism. This is a potential explanation for the superior effect of this antibiotic effect in PSC. While the other antibiotics used in the studies included here are widely considered to be "broad-spectrum," the relatively narrow specificity of vancomycin might be a potential 

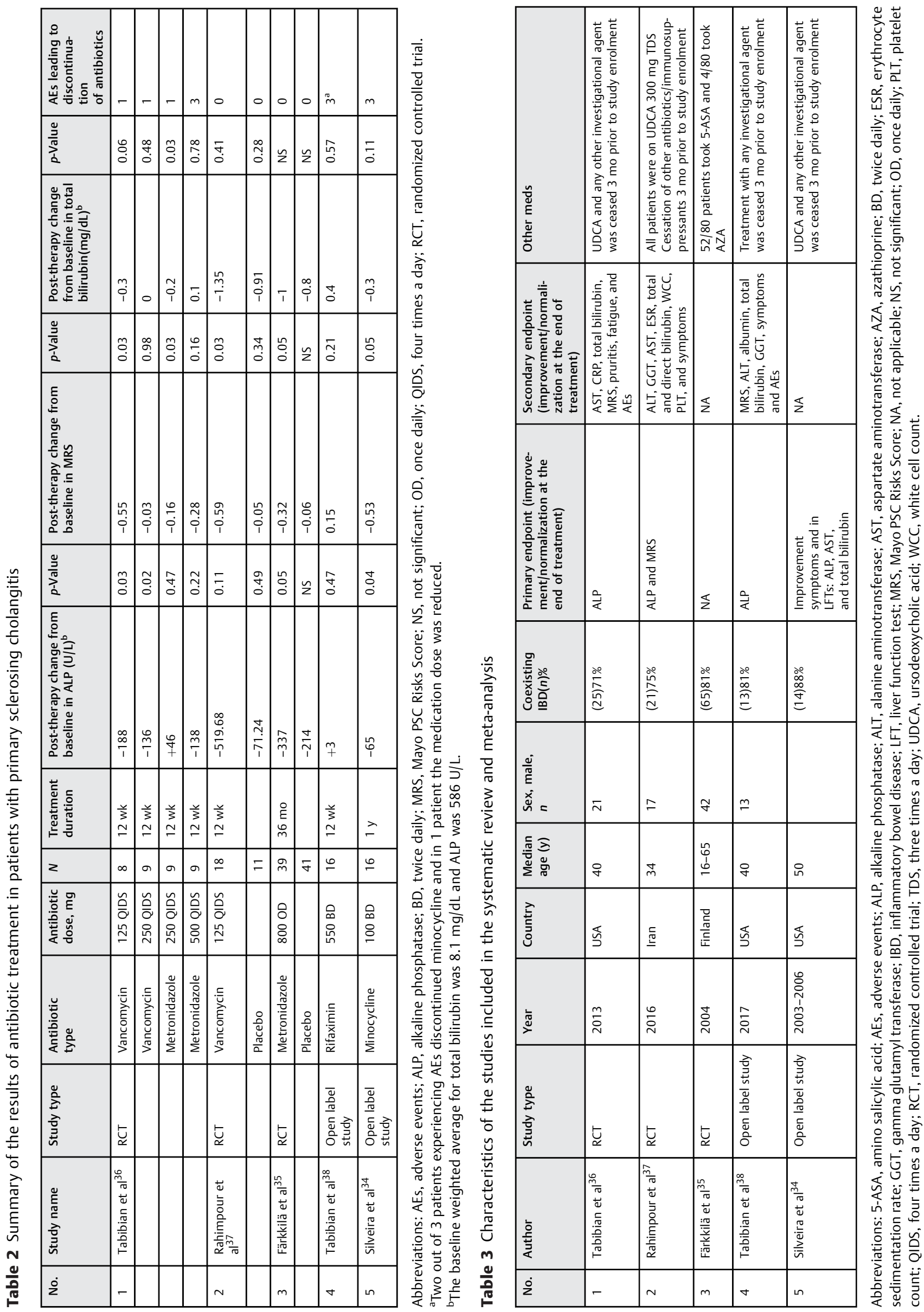

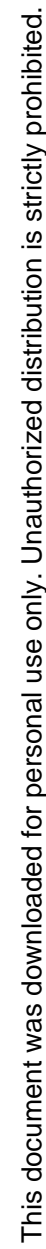




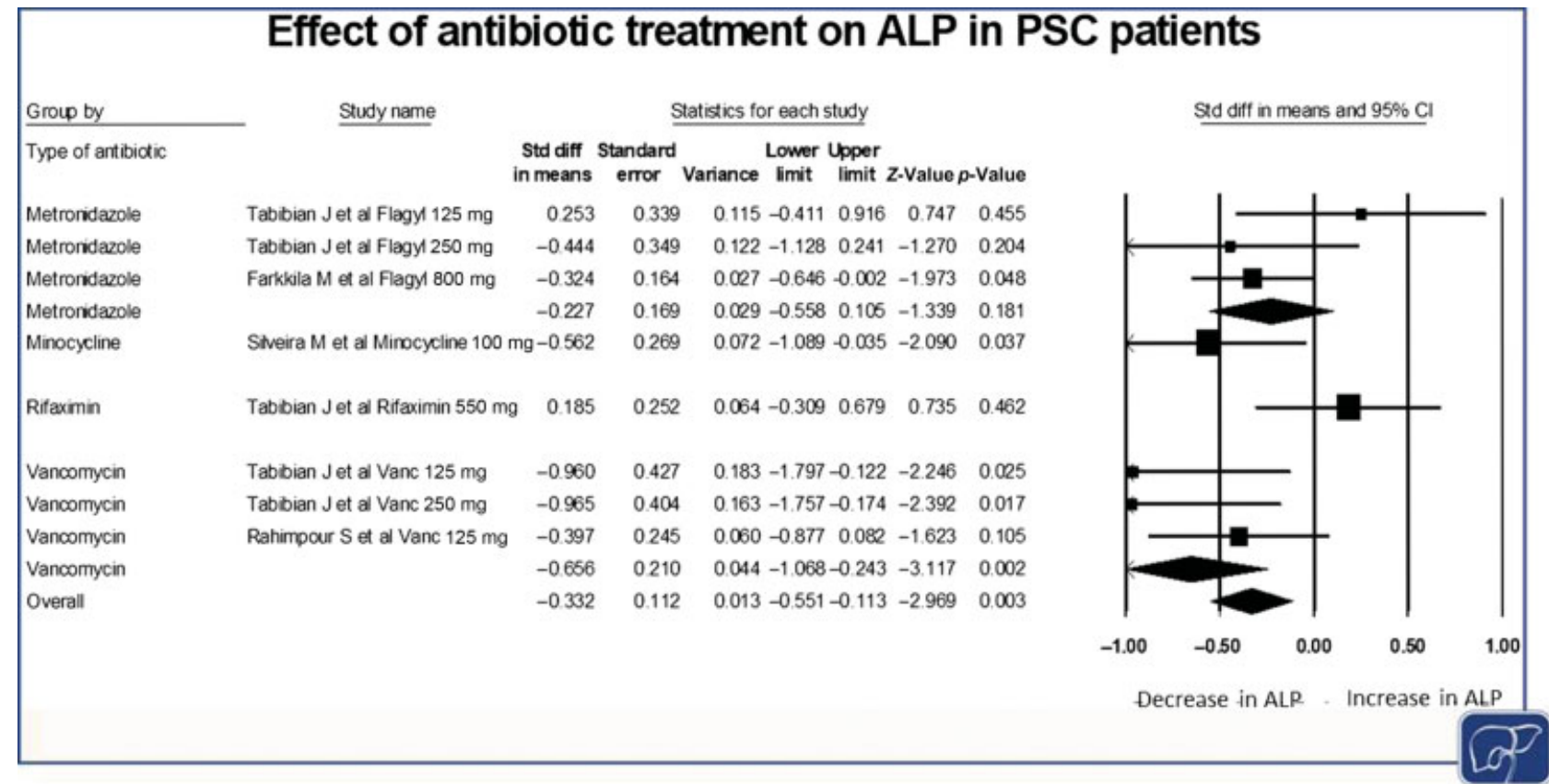

Fig. 2 Forest plot showing the change in alkaline phosphatase (ALP) postantibiotic treatment in primary sclerosing cholangitis (PSC) patients $\left(I_{2}=44.93, p=0.08\right)$. Cl, confidence interval.

\section{Effect of antibiotic treatment on MRS in PSC patients}

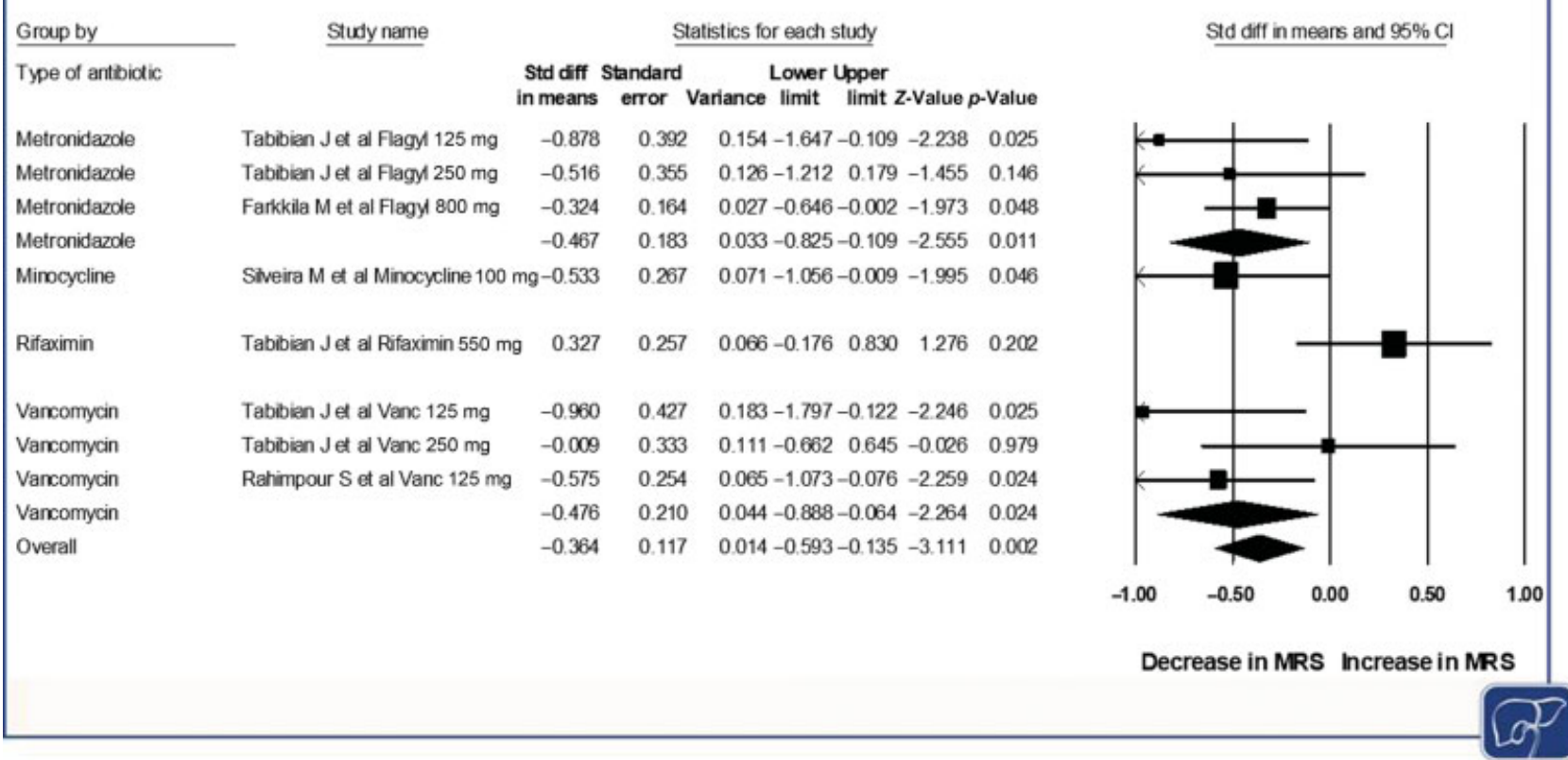

Fig. 3 Forrest plot showing the change in Mayo PSC risk score (MRS) postantibiotic treatment in primary sclerosing cholangitis (PSC) patients $\left(I_{2}=47.60, p=0.06\right)$. Cl, confidence interval.

explanation for its superior effects on PSC clinical and biochemical measures.

In an open-labeled pilot study, ${ }^{51} 14$ children with PSC and UC treated with oral vancomycin, had significant improvement or normalization in liver function tests (LFTs) (alanine aminotransferase [ALT] and gamma glutamyl transferase [GGT]), erythrocyte sedimentation rate, and clinical symptoms, with a maximal response observed in those subjects who were not cirrhotic at the start of the treatment. Wor- sening of LFTs (ALT and GGT) and recurrence of clinical symptoms occurred after treatment was discontinued. Retreatment, however, again resulted in the normalization of ALT and GGT. Autoantibodies (antinuclear antibody, antismooth muscle antibody, perinuclear antineutrophil cytoplasmic antibodies) present in 4 children became negative or improved significantly while on treatment with oral vancomycin may have an influence on immunoreactivity in PSC. A subsequent study ${ }^{33}$ of the same cohort showed in addition to 


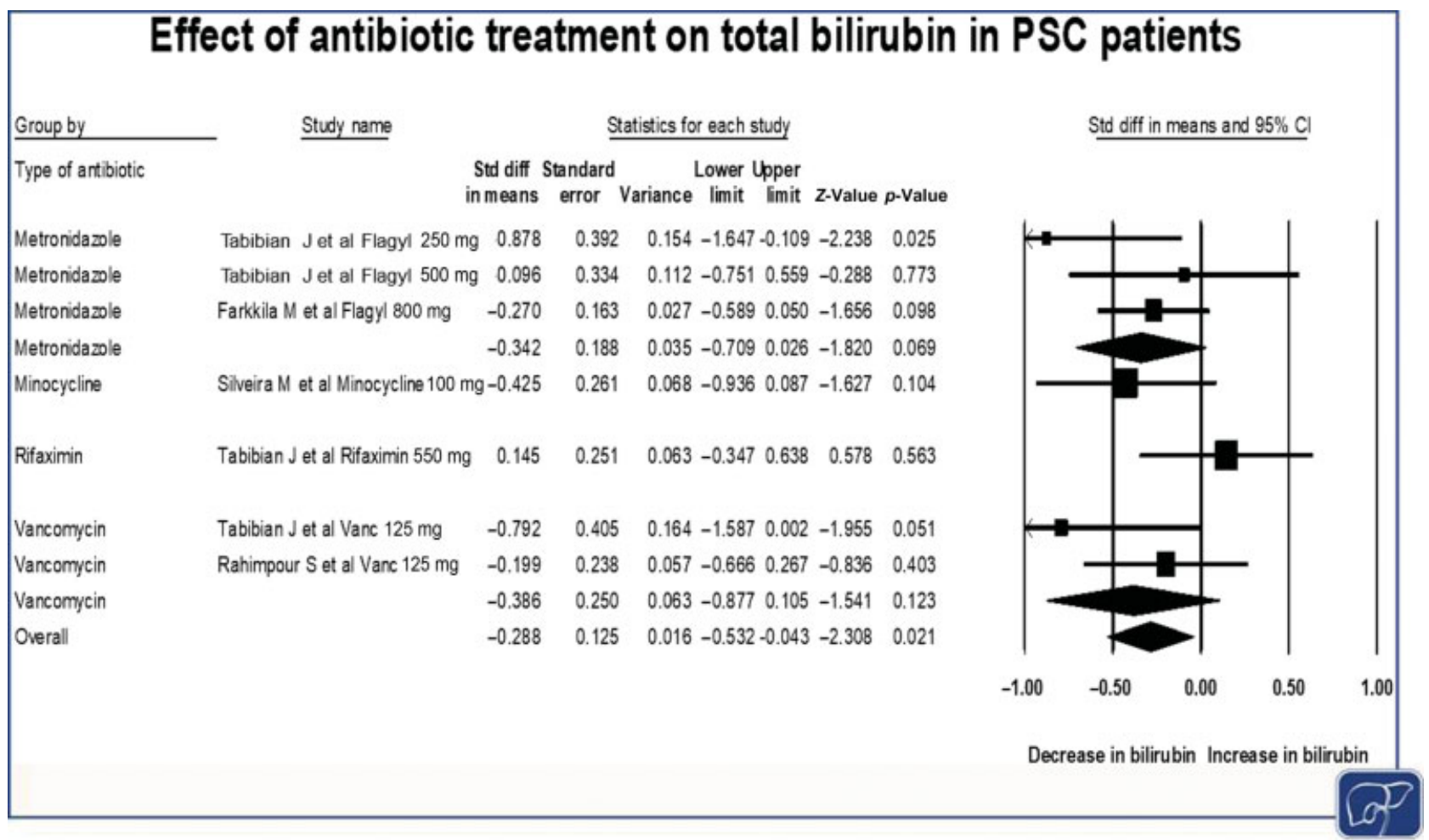

Fig. 4 Forrest plot showing the change in total bilirubin postantibiotic treatment in primary sclerosing cholangitis (PSC) patients $\left(I_{2}=20.02\right.$, $p=0.23)$. $\mathrm{Cl}$, confidence interval.

symptom resolution and normalization of liver biochemistry an increase in peripheral $\mathrm{CD} 4^{+} \mathrm{FoxP}^{+}$regulatory $\mathrm{T}$ (Tregs) cells and transforming growth factor $\beta$ levels in response to the antibiotic, suggesting an immunomodulatory effect of vancomycin. The immunomodulatory effect of vancomycin was thought to be due to its direct effect on the $\mathrm{T}$ cell inflammatory pathway via the tumor necrosis factor (TNF)- $\alpha$ pathways and downstream Treg induction. This may suggest that vancomycin has a direct or indirect immunomodulatory effect, via the TNF- $\alpha$ inflammatory pathways and/or downstream Treg induction.

Oral vancomycin has also been effective for the treatment of recurrent PSC after orthotopic liver transplant ${ }^{28}$, suggesting that the disease mechanism is not confined to the liver and involves the intestine, for example, the backflow of gut bacteria or gut bacteremia. Thus, it can be hypothesized that oral vancomycin not only specifically suppresses Gram-positive bacteria involved with primary bile acid metabolism but might also be directly or indirectly involved with attenuating the inflammatory response underpinning periportal inflammation and hepatic damage.

Metronidazole is the second most commonly studied antibiotic for the treatment of PSC and associated IBD, and two RCTs (one placebo controlled and one uncontrolled) have evaluated the efficacy and safety of metronidazole comparing it with ursodeoxycholic acid or vancomycin. Compared with vancomycin, metronidazole had numerically higher number of AEs leading to treatment discontinuation and only a very modest effect on improving ALP. On the other hand, minocycline, a semisynthetic second-generation tetracycline, studied in a single open-labeled trial had a good safety profile and was most effective among the antibiotics in improving the total serum bilirubin and MRS and its efficacy in improving serum ALP was similar to that observed for vancomycin. As such, minocycline also appears to be promising, especially given its known immunomodulatory properties independent of its antibacterial effects. ${ }^{52-54}$ In contrast, it is noteworthy that the single study evaluating rifaximin included in this meta-analysis actually failed to have any beneficial effect in PSC patients. While the antimicrobial spectrum of rifaximin is considered to be very broad, its systemic effects are limited, unlike minocycline. Taken together, despite the broad-spectrum antimicrobial effects attributed to these different antibiotics, their capacity to either directly affect host immune pathways and inflammation, as well as indirectly affect host immune and inflammatory response via their antimicrobial actions, needs greater evaluation via larger RCTs and may not target gut bacteria.

The strengths of our study include the comprehensive literature search and a rigorous statistical approach. We pooled the data with a random effects model, which provides a more conservative estimate of the effect of antibiotics on various outcome measures in PSC. We also performed subgroup analyses to assess treatment effect of individual antibiotics on primary and secondary outcome measures. However, this systematic review and meta-analysis is not without limitations. First, there is a paucity of large placebo-controlled clinical trials evaluating the efficacy of antibiotics in PSC, largely due to the rarity of PSC. Most published studies are case reports, case series, and uncontrolled trials. Only two of the three RCTs are placebo- 
controlled, limiting the conclusions that can be drawn from this review. Moreover, until recently the majority of studies assessing the impact of antimicrobial therapy in PSC were reported in the pediatric PSC populations. There was also moderate heterogeneity among the five studies included in our primary analysis, suggesting that the response to antibiotic therapy may be overestimated and could be influenced by a variety of factors. Importantly, the long-term safety profile of the antibiotics used remains unknown, but it is possible that we overestimated the rate of AEs as some of these may have been due to the underlying disease itself rather than being related to the study medication.

All trials included in this systematic review and metaanalysis have evaluated surrogate markers of PSC progression including MRS and improvement in ALP. In clinical practice, the natural history and prognosis of PSC in a patient can be established by tracking of MRS. The MRS, in turn, is used to predict survival up to 4 years. ${ }^{55}$ Any reduction in MRS could theoretically translate into an improvement in the natural course and the prognosis of a patient with PSC. Normalization of ALP is often used as a surrogate marker in clinical trials and is associated with improved long-term survival and decreased risk of requiring liver transplantation in patients with PSC. ${ }^{55-57}$ The degree to which a surrogate marker can simulate more definite endpoints, such as survival free of liver transplantation, is unclear and further studies are needed. ${ }^{58}$

This systematic review and meta-analysis suggest that antibiotics are an effective treatment option for PSC with IBD. However, the exact mechanism(s) of action of antibiotic therapy remains to be elucidated. In addition to antimicrobial and (subsequent) anti-inflammatory effects of antibiotics, almost certainly alter the gut microbiome. Several antibiotics have been used so far without major adverse side effects; however, antibiotic drug resistance always remains a concern. A recent case series ${ }^{59}$ describing use of oral vancomycin to treat colitis associated with PSC in 17 children did not demonstrate the emergence of any vancomycin-resistant enterococcus that was routinely screened for in this small cohort. Vancomycin seems to be the most promising antibacterial pharmacotherapy for PSC that has been evaluated in RCTs. However, most studies in this systematic review and meta-analysis are limited by the sample sizes of the respective clinical trials. This is due to the low prevalence and incidence of PSC.

While this systematic review supports the notion that antibiotic therapy appears to be effective in improving disease activity in PSC patients, the ideal antibiotic, dose, and regimen remain largely unknown, and the use of definite primary endpoints rather than surrogate markers to define the natural history of PSC needs to be studied. Thus, adequately powered placebo-controlled RCTs are needed with a longer treatment duration and follow-up period to evaluate the effect of variable doses of vancomycin on more definite endpoints including cholangiography findings and liver transplant-free survival. In addition, state-of-the-art molecular technologies should be applied to properly explore the interdependence between the gas- trointestinal microbiome, immune system, genetic profile, and response to antibiotic therapy.

Writing Assistance

None.

\section{Authors' Contributions}

Ayesha Shah and Gerald Holtmann: Study idea, concept and design, data extraction and interpretation of data, and drafting of the manuscript.

Darrel Crawford: Analysis and interpretation of data, input into the manuscript, and review of final manuscript. Daniel Burger: Input into discussions and review of final manuscript.

Mark Morrison: Study idea, concept and design, drafting of the manuscript, and review of final manuscript.

Mike Jones: Data analysis and review of final manuscript. Nicholas Talley: Study idea, data interpretation, drafting of the manuscript and review of final manuscript.

Peter Lewindon: Drafting of the manuscript and review of final manuscript.

Marjorie Walker: Drafting of the manuscript and review of final manuscript.

Katherine Stuart: Drafting of the manuscript and review of final manuscript.

Graeme Macdonald: Drafting of the manuscript and review of final manuscript.

Neal Martin: Drafting of the manuscript and review of final manuscript.

Caroline Tallis: Drafting of the manuscript and review of final manuscript.

Simon Keely: Drafting of the manuscript and review of final manuscript.

Funding

None.

Conflict of Interest

None declared.

Acknowledgments

None.

\section{References}

1 Lee YM, Kaplan MM. Primary sclerosing cholangitis. N Engl J Med 1995;332(14):924-933

2 Wiesner RH, Grambsch PM, Dickson ER, et al. Primary sclerosing cholangitis: natural history, prognostic factors and survival analysis. Hepatology 1989;10(04):430-436

3 Broomé U, Olsson R, Lööf L, et al. Natural history and prognostic factors in 305 Swedish patients with primary sclerosing cholangitis. Gut 1996;38(04):610-615

4 Farrant JM, Hayllar KM, Wilkinson ML, et al. Natural history and prognostic variables in primary sclerosing cholangitis. Gastroenterology 1991;100(06):1710-1717

5 Bambha K, Kim WR, Talwalkar J, et al. Incidence, clinical spectrum, and outcomes of primary sclerosing cholangitis in a United States community. Gastroenterology 2003;125(05): 1364-1369 
6 Boonstra K, Beuers U, Ponsioen CY. Epidemiology of primary sclerosing cholangitis and primary biliary cirrhosis: a systematic review. J Hepatol 2012;56(05):1181-1188

7 Toy E, Balasubramanian S, Selmi C, Li CS, Bowlus CL. The prevalence, incidence and natural history of primary sclerosing cholangitis in an ethnically diverse population. BMC Gastroenterol 2011;11:83

8 Olsson R, Danielsson A, Järnerot G, et al. Prevalence of primary sclerosing cholangitis in patients with ulcerative colitis. Gastroenterology 1991;100(5 Pt 1):1319-1323

9 Fausa O, Schrumpf E, Elgjo K. Relationship of inflammatory bowel disease and primary sclerosing cholangitis. Semin Liver Dis 1991; 11(01):31-39

10 Loftus EV Jr, Sandborn WJ, Lindor KD, Larusso NF. Interactions between chronic liver disease and inflammatory bowel disease. Inflamm Bowel Dis 1997;3(04):288-302

11 Eaton JE, Talwalkar JA, Lazaridis KN, Gores GJ, Lindor KD. Pathogenesis of primary sclerosing cholangitis and advances in diagnosis and management. Gastroenterology 2013;145(03): 521-536

12 Gasbarrini A, Corazza GR, Gasbarrini G, et al; 1st Rome H2-Breath Testing Consensus Conference Working Group. Methodology and indications of H2-breath testing in gastrointestinal diseases: the Rome Consensus Conference. Aliment Pharmacol Ther 2009;29 (Suppl 1):1-49

13 Chapman R, Fevery J, Kalloo A, et al; American Association for the Study of Liver Diseases. Diagnosis and management of primary sclerosing cholangitis. Hepatology 2010;51(02):660-678

14 Boonstra K, Weersma RK, van Erpecum KJ, et al; EpiPSCPBC Study Group. Population-based epidemiology, malignancy risk, and outcome of primary sclerosing cholangitis. Hepatology 2013;58 (06):2045-2055

15 Visseren T, Darwish Murad S. Recurrence of primary sclerosing cholangitis, primary biliary cholangitis and auto-immune hepatitis after liver transplantation. Best Pract Res Clin Gastroenterol 2017;31(02):187-198

16 Lindor KD, Kowdley KV, Harrison ME; American College of Gastroenterology. ACG clinical guideline: primary sclerosing cholangitis. Am J Gastroenterol 2015;110(05):646-659, quiz 660

17 Rankin JG, Boden RW, Goulston SJ, Morrow W. The liver in ulcerative colitis; treatment of pericholangitis with tetracycline. Lancet 1959;2(7112):1110-1112

18 Cheung AC, Lazaridis KN, LaRusso NF, Gores GJ. Emerging pharmacologic therapies for primary sclerosing cholangitis. Curr Opin Gastroenterol 2017;33(03):149-157

19 Tabibian JH, O'Hara SP, Splinter PL, Trussoni CE, LaRusso NF. Cholangiocyte senescence by way of N-ras activation is a characteristic of primary sclerosing cholangitis. Hepatology 2014;59 (06):2263-2275

20 Tabibian JH, Trussoni CE, O'Hara SP, Splinter PL, Heimbach JK, LaRusso NF. Characterization of cultured cholangiocytes isolated from livers of patients with primary sclerosing cholangitis. Lab Invest 2014;94(10):1126-1133

21 Kono K, Ohnishi K, Omata M, et al. Experimental portal fibrosis produced by intraportal injection of killed nonpathogenic Escherichia coli in rabbits. Gastroenterology 1988;94(03):787796

22 Lichtman SN, Okoruwa EE, Keku J, Schwab JH, Sartor RB. Degradation of endogenous bacterial cell wall polymers by the muralytic enzyme mutanolysin prevents hepatobiliary injury in genetically susceptible rats with experimental intestinal bacterial overgrowth. J Clin Invest 1992;90(04):1313-1322

23 Hobson CH, Butt TJ, Ferry DM, Hunter J, Chadwick VS, Broom MF. Enterohepatic circulation of bacterial chemotactic peptide in rats with experimental colitis. Gastroenterology 1988;94(04): 1006-1013

24 Karlsen TH. Primary sclerosing cholangitis: 50 years of a gut-liver relationship and still no love? Gut 2016;65(10):1579-1581
25 Torres J, Palmela C, Brito H, et al. The gut microbiota, bile acids and their correlation in primary sclerosing cholangitis associated with inflammatory bowel disease. United European Gastroenterol J 2018;6(01):112-122

26 Tabibian JH, Ali AH, Lindor KD. Primary sclerosing cholangitis, part 1: epidemiology, etiopathogenesis, clinical features, and treatment. Gastroenterol Hepatol (N Y) 2018;14(05):293-304

27 Buness C, Lindor KD, Miloh T. Oral vancomycin therapy in a child with primary sclerosing cholangitis and severe ulcerative colitis. Pediatr Gastroenterol Hepatol Nutr 2016;19(03):210-213

28 Davies YK, Tsay CJ, Caccamo DV, Cox KM, Castillo RO, Cox KL. Successful treatment of recurrent primary sclerosing cholangitis after orthotopic liver transplantation with oral vancomycin. Case Rep Transplant 2013;2013:314292

29 Kozaiwa K, Tajiri H, Sawada A, et al. Three paediatric cases of primary sclerosing cholangitis treated with ursodeoxycholic acid and sulphasalazine. J Gastroenterol Hepatol 1998;13(08):825-829

30 Boner AL, Peroni D, Bodini A, Delaini G, Piacentini G. Azithromycin may reduce cholestasis in primary sclerosing cholangitis: a case report and serendipitous observation. Int J Immunopathol Pharmacol 2007;20(04):847-849

31 Tada S, Ebinuma H, Saito H, Hibi T. Therapeutic benefit of sulfasalazine for patients with primary sclerosing cholangitis. J Gastroenterol 2006;41(04):388-389

32 Cox KL, Cox KM. Oral vancomycin: treatment of primary sclerosing cholangitis in children with inflammatory bowel disease. J Pediatr Gastroenterol Nutr 1998;27(05):580-583

33 Abarbanel DN, Seki SM, Davies Y, et al. Immunomodulatory effect of vancomycin on Treg in pediatric inflammatory bowel disease and primary sclerosing cholangitis. J Clin Immunol 2013;33(02): 397-406

34 Silveira MG, Torok NJ, Gossard AA, et al. Minocycline in the treatment of patients with primary sclerosing cholangitis: results of a pilot study. Am J Gastroenterol 2009;104(01):83-88

35 Färkkilä M, Karvonen AL, Nurmi H, et al. Metronidazole and ursodeoxycholic acid for primary sclerosing cholangitis: a randomized placebo-controlled trial. Hepatology 2004;40(06):1379-1386

36 Tabibian JH, Weeding E, Jorgensen RA, et al. Randomised clinical trial: vancomycin or metronidazole in patients with primary sclerosing cholangitis - a pilot study. Aliment Pharmacol Ther 2013;37(06):604-612

37 Rahimpour S, Nasiri-Toosi M, Khalili H, Ebrahimi-Daryani N, Nouri-Taromlou MK, Azizi Z. A triple blinded, randomized, placebo-controlled clinical trial to evaluate the efficacy and safety of oral vancomycin in primary sclerosing cholangitis: a pilot study. J Gastrointestin Liver Dis 2016;25(04):457-464

38 Tabibian JH, Gossard A, El-Youssef M, et al. Prospective clinical trial of rifaximin therapy for patients with primary sclerosing cholangitis. Am J Ther 2017;24(01):e56-e63

39 Rupp C, Rössler A, Halibasic E, et al. Reduction in alkaline phosphatase is associated with longer survival in primary sclerosing cholangitis, independent of dominant stenosis. Aliment Pharmacol Ther 2014;40(11-12):1292-1301

40 Kim WR, Therneau TM, Wiesner RH, et al. A revised natural history model for primary sclerosing cholangitis. Mayo Clin Proc 2000;75(07):688-694

41 Stroup DF, Berlin JA, Morton SC, et al. Meta-analysis of observational studies in epidemiology: a proposal for reporting. Metaanalysis Of Observational Studies in Epidemiology (MOOSE) group. JAMA 2000;283(15):2008-2012

42 Moher D, Liberati A, Tetzlaff J, Altman DG; PRISMA Group. Preferred reporting items for systematic reviews and meta-analyses: the PRISMA statement. BMJ 2009;339:b2535

43 Higgins JP, Thompson SG. Quantifying heterogeneity in a metaanalysis. Stat Med 2002;21(11):1539-1558

44 DerSimonian R, Laird N. Meta-analysis in clinical trials. Control Clin Trials 1986;7(03):177-188 
45 Ali AH, Carey EJ, Lindor KD. Current research on the treatment of primary sclerosing cholangitis. Intractable Rare Dis Res 2015;4(01): $1-6$

46 Vrieze A, Out C, Fuentes S, et al. Impact of oral vancomycin on gut microbiota, bile acid metabolism, and insulin sensitivity. J Hepatol 2014;60(04):824-831

47 Begley M, Hill C, Gahan CG. Bile salt hydrolase activity in probiotics. Appl Environ Microbiol 2006;72(03):1729-1738

48 Kitahara M, Sakata S, Sakamoto M, Benno Y. Comparison among fecal secondary bile acid levels, fecal microbiota and Clostridium scindens cell numbers in Japanese. Microbiol Immunol 2004;48 (05):367-375

49 Low-Beer TS, Nutter S. Colonic bacterial activity, biliary cholesterol saturation, and pathogenesis of gallstones. Lancet 1978;2 (8099):1063-1065

50 Ajouz H, Mukherji D, Shamseddine A. Secondary bile acids: an underrecognized cause of colon cancer. World J Surg Oncol 2014; 12:164-164

51 Davies YK, Cox KM, Abdullah BA, Safta A, Terry AB, Cox KL. Longterm treatment of primary sclerosing cholangitis in children with oral vancomycin: an immunomodulating antibiotic. J Pediatr Gastroenterol Nutr 2008;47(01):61-67

52 Ledeboer A, Sloane EM, Milligan ED, et al. Minocycline attenuates mechanical allodynia and proinflammatory cytokine expression in rat models of pain facilitation. Pain 2005;115(1-2):71-83
53 Van den Bogert C, Kroon AM. Effects of oxytetracycline on in vivo proliferation and differentiation of erythroid and lymphoid cells in the rat. Clin Exp Immunol 1982;50(02):327-335

54 Sewell KL, Breedveld F, Furrie E, et al. The effect of minocycline in rat models of inflammatory arthritis: correlation of arthritis suppression with enhanced T cell calcium flux. Cell Immunol 1996;167(02):195-204

55 Stanich PP, Björnsson E, Gossard AA, Enders F, Jorgensen R, Lindor KD. Alkaline phosphatase normalization is associated with better prognosis in primary sclerosing cholangitis. Dig Liver Dis 2011;43 (04):309-313

56 Lindor KD, Kowdley KV, Luketic VA, et al. High-dose ursodeoxycholic acid for the treatment of primary sclerosing cholangitis. Hepatology 2009;50(03):808-814

57 Hilscher M, Enders FB, Carey EJ, Lindor KD, Tabibian JH. Alkaline phosphatase normalization is a biomarker of improved survival in primary sclerosing cholangitis. Ann Hepatol 2016;15(02): 246-253

58 Silveira MG, Lindor KD. High dose ursodeoxycholic acid for the treatment of primary sclerosing cholangitis. J Hepatol 2008;48 (05):692-694

59 Tan LZ, Reilly CR, Steward-Harrison LC, Balouch F, Muir R, Lewindon PJ. Oral vancomycin induces clinical and mucosal remission of colitis in children with primary sclerosing cholangitis-ulcerative colitis. Gut 2018:gutjnl-2018-316599 